\title{
Long non-coding RNA small nucleolar RNA host gene 7 is upregulated and promotes cell proliferation in thyroid cancer
}

\author{
LI CHEN $^{1 *}$, JING ZHU ${ }^{2 *}$ and LING-JIE ZHANG ${ }^{3}$ \\ Departments of ${ }^{1}$ Endocrinology and ${ }^{2}$ Clinical Laboratory, Jingzhou Central Hospital, \\ The Second Clinical Medical College, Yangtze University, Jingzhou, Hubei 434020; ${ }^{3}$ Department of Anesthesiology, \\ Hubei Provincial Hospital of Integrated Chinese and Western Medicine, Wuhan, Hubei 430015, P.R. China
}

Received September 12, 2019; Accepted June 6, 2019

DOI: $10.3892 / 01.2019 .10782$

\begin{abstract}
Thyroid cancer (THCA) is one of the most common types of endocrine cancer worldwide. However, the mechanisms underlying THCA progression have not been fully elucidated. Recent studies have demonstrated that long non-coding RNAs (IncRNAs) are dysregulated in human diseases, and are involved in regulating various biological processes. Furthermore, several reports have indicated that lncRNAs serve important roles in THCA. In the present study, a dataset from The Cancer Genome Atlas was used to analyze the expression levels and the clinical information of small nucleolar RNA host gene 7 (SNHG7) in THCA. Starbase was used to construct the competing endogenous RNA network. The Molecule Annotation System was used to analyze the data from Gene Ontology and Kyoto Encyclopedia of Genes and Genomes databases. Furthermore, cell proliferation and cell cycle assays were used to detect the functions of SNHG7 in THCA. The present study revealed for the first time, to the best of our knowledge, that SNHG7 is markedly upregulated in THCA samples following analysis of The Cancer Genome Atlas datasets. SNHG7 expression was higher in advanced stage compared with early stage THCA samples. In addition, high expression levels of SNHG7 were associated with shorter survival times in THCA patients compared with low expression
\end{abstract}

Correspondence to: Professor Ling-Jie Zhang, Department of Anesthesiology, Hubei Provincial Hospital of Integrated Chinese and Western Medicine, 11 Lingjiaohu Road, Jianghan, Wuhan, Hubei 430015, P.R. China

E-mail: 22457419@qq.com

*Contributed equally

Abbreviations: ER, endoplasmic reticulum; lncRNAs, long non-coding RNAs; TNM, Tumor-Node-Metastasis; DFS, disease-free survival; SNHG7, small nucleolar RNA host gene 7; THCA, thyroid cancer; TCGA, The Cancer Genome Atlas; ceRNA, competing endogenous RNA

Key words: thyroid cancer, small nucleolar RNA host gene 7, prognostic target, proliferation, long non-coding RNA levels. Bioinformatics analysis revealed that SNHG7 was associated with the processes of 'protein translation', 'viral life cycle', 'RNA processing', 'mRNA splicing', 'histone ubiquitination', 'endoplasmic reticulum-to-Golgi vesicle-mediated transport', 'sister chromatid cohesion', 'DNA damage checkpoint regulation', 'translation' and 'the spliceosome'. Additionally, knockdown of SNHG7 significantly inhibited thyroid cancer cell proliferation and cell cycle progression in vitro. Taken together, the results obtained in the present study suggested that SNHG7 may serve as a novel therapeutic and prognostic target for THCA.

\section{Introduction}

Thyroid cancer (THCA) is one of the most common types of endocrine cancer worldwide $(1,2)$. Accumulating evidence has indicated that numerous biomarkers are involved in THCA prognosis, proliferation and radiation resistance. For example, Huang et al (3) observed that downregulation of microRNA (miR)-125a-5p promoted glucose metabolism by upregulating CD147 in THCA. Furthermore, Choi et al (4) demonstrated that prospero homeobox 1 activates $\mathrm{Wnt} / \beta$-catenin signaling, and induces the expression of serpin family A member 1 and fatty acid binding protein 4 in papillary THCA. However, the mechanisms underlying THCA progression have not been fully elucidated.

Long noncoding RNAs (IncRNAs) are a type of RNA transcript that are $>200$ bp in length, but which have no protein coding potential (5). Previous studies have revealed that lncRNAs are dysregulated in a number of human diseases, and are involved in regulating various biological processes, including RNA stabilization $(6,7)$, X chromosome inactivation $(8,9)$, transcription and protein translation (10). Several reports have described how lncRNAs serve important roles in THCA. For example, a recent study demonstrated that nuclear-enriched abundant transcript $1 \_2$ is able to 'sponge' (or competitively inhibit) miR-106b-5p to promote the expression of ATPase family AAA domain containing 2 in papillary THCA (11). The lncRNA CNALPTC1 acts as a competing endogenous RNA (ceRNA) by sponging the miR-30 family in THCA (12). Several studies have demonstrated that lncRNA expression is associated with the prognosis of patients with THCA (13-15). Li et al (16) identified 111 IncRNAs that were 
differentially expressed between tumor and normal samples in THCA. Furthermore, the lncRNA growth arrest specific 5 was significantly associated with Tumor-Node-Metastasis (TNM) staging, lymph node metastasis, disease-free survival (DFS) and overall survival in THCA (17).

Small nucleolar RNA host gene 7 (SNHG7) is a novel lncRNA involved in the progression of different types of human cancer. IncRNA SNHG7 was first reported to be an oncogenic gene in lung cancer, increasing the expression of Fas apoptotic inhibitory molecule 2 (18). Wang et al (19) demonstrated that lncRNA SNHG7 promotes the proliferation and inhibits apoptosis of gastric cancer cells by repressing P15 and P16 expression. However, the clinical value and functional role of SNHG7 in THCA has not been fully elucidated. The present study investigated the expression pattern and molecular functions of SNHG7 in THCA.

\section{Materials and methods}

Analysis of The Cancer Genome Atlas (TCGA) and Gene Expression Omnibus (GEO) datasets. The public TCGA (cancergenome.nih.gov) database was used to analyze the expression levels of SNHG7 in THCA. The clinical information of the THCA TCGA dataset was generated from cBioPortal (www.cbioportal.org) (20,21). The 2009 TNM classification of the American Joint Committee on Cancer/International Union Against Cancer was used to stage all the patients (22). The survival analysis, comparing patients with THCA with a high expression level of SNHG7 (SNHG7-high) and those with a low expression level of SNHG7 (SNHG7-low), was performed. In the present study, the web application Cutoff Finder 2012 (molpath. charite.de/cutoff) was used to identify the cut-off value (median expression value) to divide the THCA samples into SNHG7-high and SNHG7-low groups. In order to validate TCGA analysis, two GEO datasets, GSE50901, samples obtained from adjacent healthy tissues from patients with THCA (23), and GSE33630, obtained from healthy controls $(24,25)$, were analyzed. The normalized data were downloaded from the GEO repository (https://www.ncbi. nlm.nih.gov/gds). The normal samples in the TCGA and GSE50901 datasets were adjacent normal tissue samples taken from patients with thyroid cancer. The normal samples in GSE33630 were taken from control patients.

Construction of SNHG7-mediated ceRNA networks. In the present study, SNHG7- targeting microRNAs and mRNAs were predicted using Starbase version 3.0 (http://starbase. sysu.edu.cn/index.php). Subsequently, co-expression analysis was performed for SNHG7 in the TCGA THCA dataset using the cBioPortal online software. The SNHG7-target pairs with co-expression coefficient $>0.3$ were selected for the construction of SNHG7-associated ceRNA networks. The Cytoscape software version 3.4.0 (www.cytoscape.org) was used for visualization of the co-expression networks.

Co-expression network construction and analysis. According to the expression value of the Pearson's correlation coefficient of SNHG7-gene pairs, SNHG7 co-expressed genes were identified in the present study. The co-expressed SNHG7-gene pairs with an absolute value of Pearson's correlation coefficient $\geq 0.3$ were selected, which included the top 1,000 genes that were co-expressed with SNHG7 in thyroid cancer samples, and the co-expression network was established by using the Cytoscape software.

Protein-protein interaction (PPI) network construction and analysis. By using the Search Tool for the Retrieval of Interacting Genes/Proteins database version 11.0 (STRING \#11.0) (26), a PPI network mediated by SNHG7 was constructed using its co-expressing mRNAs (combined score, $>0.4$ ). Subsequently, a module analysis of the network was performed using the Mcode plugin (27). The PPI network with the cut-offs of degree $\geq 2$ and of nodes $\geq 2$-core were selected as hub networks.

Gene Ontology (GO) and Kyoto Encyclopedia of Genes and Genomes (KEGG) pathway analysis. In the present study, GO and KEGG pathway analyses were performed by using the Molecule Annotation System version 3.0 (bioinfo.capitalbio. com $/ \mathrm{mas} 3) . \mathrm{P}<0.05$ was considered to indicate a statistically significant difference.

Cell culture and cell transfection. The thyroid gland undifferentiated (anaplastic) carcinoma CAL62 and the thyroid gland squamous SW579 carcinoma cell lines were purchased from the American Type Culture Collection. The cells were incubated in RPMI-1640 medium (Gibco; Thermo Fisher Scientific, Inc.) supplemented with $10 \%$ fetal bovine serum (ExCell Bio) in an incubator containing $5 \% \mathrm{CO}_{2}$ at $37^{\circ} \mathrm{C}$. The small interfering (siRNA) for SNHG7 (5'-UUAGCAGAG UAAUUUGCACUU-3') and siRNA for the negative control (siNC; 5'-UAGCGACUAAACACAUCAA-3') were purchased from Guangzhou RiboBio Co., Ltd. The cells were seeded at a density of $3 \times 10^{5}$ cells/well in 6 -well plates. Following $12 \mathrm{~h}$ of incubation in an incubator containing $5 \% \mathrm{CO}_{2}$ at $37^{\circ} \mathrm{C}$, the cells were transfected with $50 \mathrm{nM}$ siNC or siSNHG7 using FuGENE $^{\circledR} 6$ transfection reagent (Promega Corporation). The transfected cells were then used to perform the cell proliferation assay and cell cycle assay.

Cell proliferation assay. Cell proliferation of the transfected cells was detected using the Cell Counting Kit-8 (CCK-8) assay. In brief, the cells were seeded at a density of 1,000 cells/well in the medium in a 96-well plate and cell proliferation was measured at $0,24,48$ and $72 \mathrm{~h}$. The cells were incubated in an incubator containing $5 \% \mathrm{CO}_{2}$ at $37^{\circ} \mathrm{C}$ with $10 \mu \mathrm{l}$ of CCK-8 solution (Dojindo Molecular Technologies, Inc.) in each well for $2 \mathrm{~h}$. The absorbance at $450 \mathrm{~nm}$ was subsequently measured using a microplate reader.

Cell cycle assay. Transfected cells were collected $48 \mathrm{~h}$ after transfection, and then the cells were fixed with pre-cooled $70 \%$ ethanol, and incubated overnight at $4^{\circ} \mathrm{C}$. Subsequently, the cells were washed once with $1 \mathrm{ml} \mathrm{PBS}$, followed by incubation in the dark with $500 \mu \mathrm{l}$ PBS containing $50 \mu \mathrm{g} / \mathrm{ml}$ propidium iodide, $0.2 \%$ Triton X-100 and $100 \mu \mathrm{g} / \mathrm{ml}$ RNase A for $30 \mathrm{~min}$ at $4^{\circ} \mathrm{C}$. The cell cycle was analyzed using a flow cytometer and the Modfit LT version 4.1 software (Verity Software House, Inc.). 

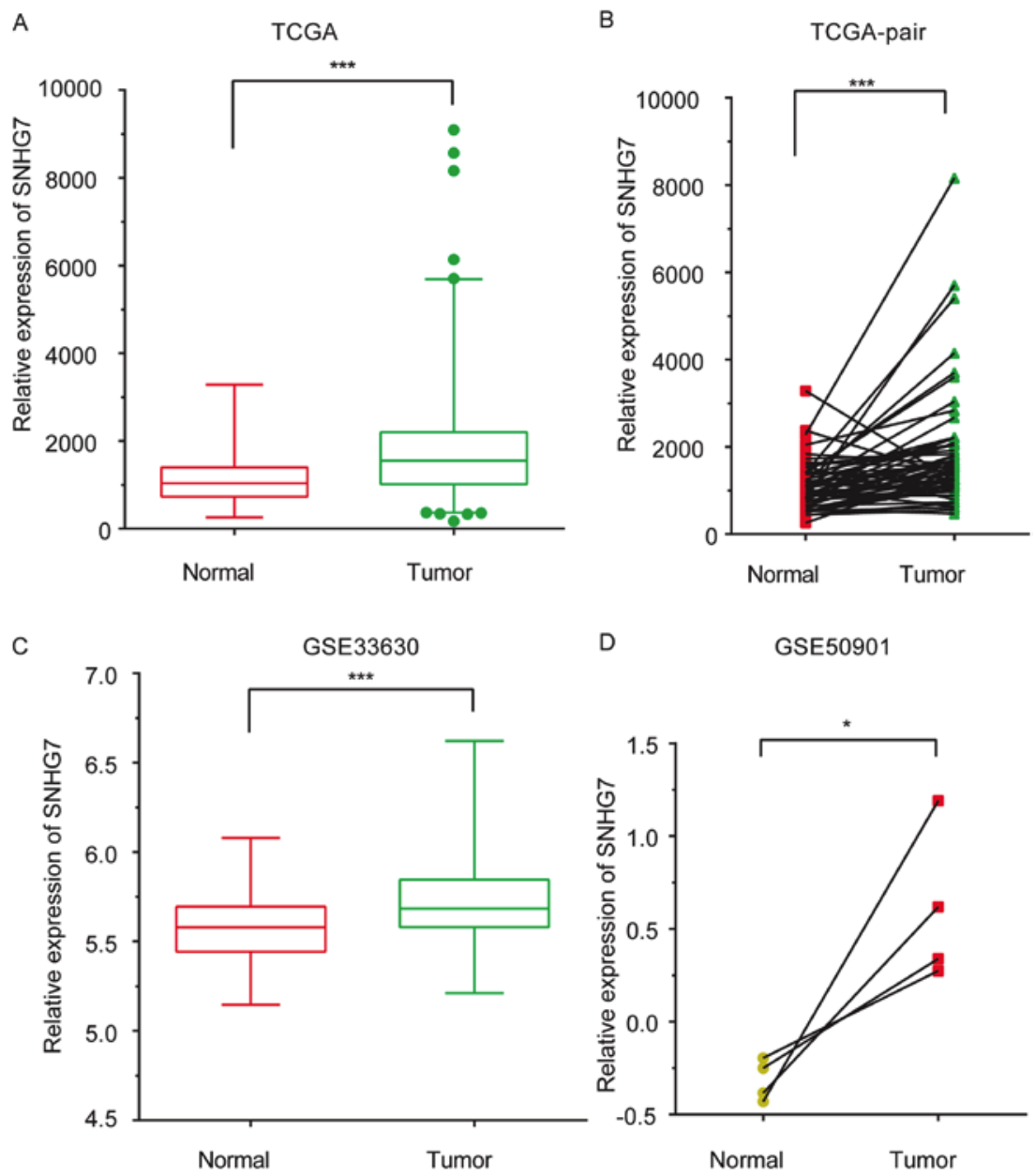

Figure 1. SNHG7 is upregulated in thyroid cancer. By analyzing (A) TCGA THCA dataset and (B) TCGA paired-THCA dataset, (C) GSE50901 and (D) GSE33630 datasets, SNHG7 was revealed to be significantly upregulated in thyroid cancer compared with normal samples. The normal samples in the TCGA THCA dataset, TCGA paired-THCA dataset and GSE50901 datasets were adjacent normal tissue samples taken from patients with thyroid cancer. The normal samples in GSE33630 were taken from control patients. Statistical comparisons between two groups in (A) and (C) were performed by Mann-Whitney $\mathrm{U}$ test. Statistical comparisons between the two paired groups in (B) and (D) were performed using the paired t-test. " $\mathrm{P}<0.05 ;{ }^{* * *} \mathrm{P}<0.001$. SNHG7, small nucleolar RNA host gene 7; TCGA, The Cancer Genome Atlas.

Reverse transcription-quantitative (RT-q)PCR assay. Total RNA was extracted using the E.Z.N.A ${ }^{\circledR}$ Total RNA kit (Omega Bio-Tek, Inc.) $48 \mathrm{~h}$ following transfection of the SW579 and CAL62 cell lines. RT was performed using the PrimeScrip RT reagent Kit (Takara Biotechnology Co., Ltd.) according to the manufacturer's instructions. Temperature conditions for the RT reaction were: $37^{\circ} \mathrm{C}$ for $15 \mathrm{~min}$; and for reverse transcriptase inactivation reaction, $85^{\circ} \mathrm{C}$ for $5 \mathrm{sec}$. RT-qPCR was performed using the Hieff qPCR SYBR-Green Master mix (YeaSen) according to the manufacturer's instructions. Temperature protocol for pre-denaturation was: $95^{\circ} \mathrm{C}$ for $5 \mathrm{~min}$; for PCR reaction ( 35 cycles), $95^{\circ} \mathrm{C}$ for $5 \mathrm{sec}$ and $60^{\circ} \mathrm{C}$ for $30 \mathrm{sec} . \beta$-actin was used as the internal control. The relative expression was calculated by the $2^{-\Delta \Delta \mathrm{Cq}}$ method (28) and every experiment was performed in triplicates. The primer sequences were as follows: SNHG7 forward, 5'-CGATACCATTGAACACGC TGC-3' andreverse, 5'-GGTTGAGGGTCCCAGTG-3'; $\beta$-actin forward, 5'-CTCCATCCTGGCCTCGCTGT-3' and reverse, 5'-GCTGTCACCTTCACCGTTCC-3'.

Statistical analysis. Statistical analyses and graphical representations were performed using SPSS version 17.0 (SPSS, Inc.) and GraphPad Prism version 6.0 (GraphPad Software, Inc.) Statistical comparisons between two groups were performed using the t-test or the Mann-Whitney U test. Statistical comparisons between two paired groups were performed by the paired t-test. For analysis of $>2$ groups, one-way analysis of variance followed by Newman-Keuls post hoc test was used. Survival curves were plotted using the Kaplan-Meier method and the differences were examined using the log-rank test. Experiments were repeated at least three times. $\mathrm{P}<0.05$ was considered to indicate a significantly significant difference.

\section{Results}

lncRNA SNHG7 is upregulated in THCA. In the present study, SNHG7 expression levels in samples from patients with THCA were compared with those in normal samples by analyzing the THCA TCGA dataset. A total of 56 normal samples and 578 tumor samples were included in the TCGA dataset. As presented in Fig. 1A and B, SNHG7 was observed to be markedly upregulated in tumor samples compared with control samples $(\mathrm{P}<0.001)$. In order to validate TCGA analysis, two GEO datasets, GSE50901 (samples obtained 

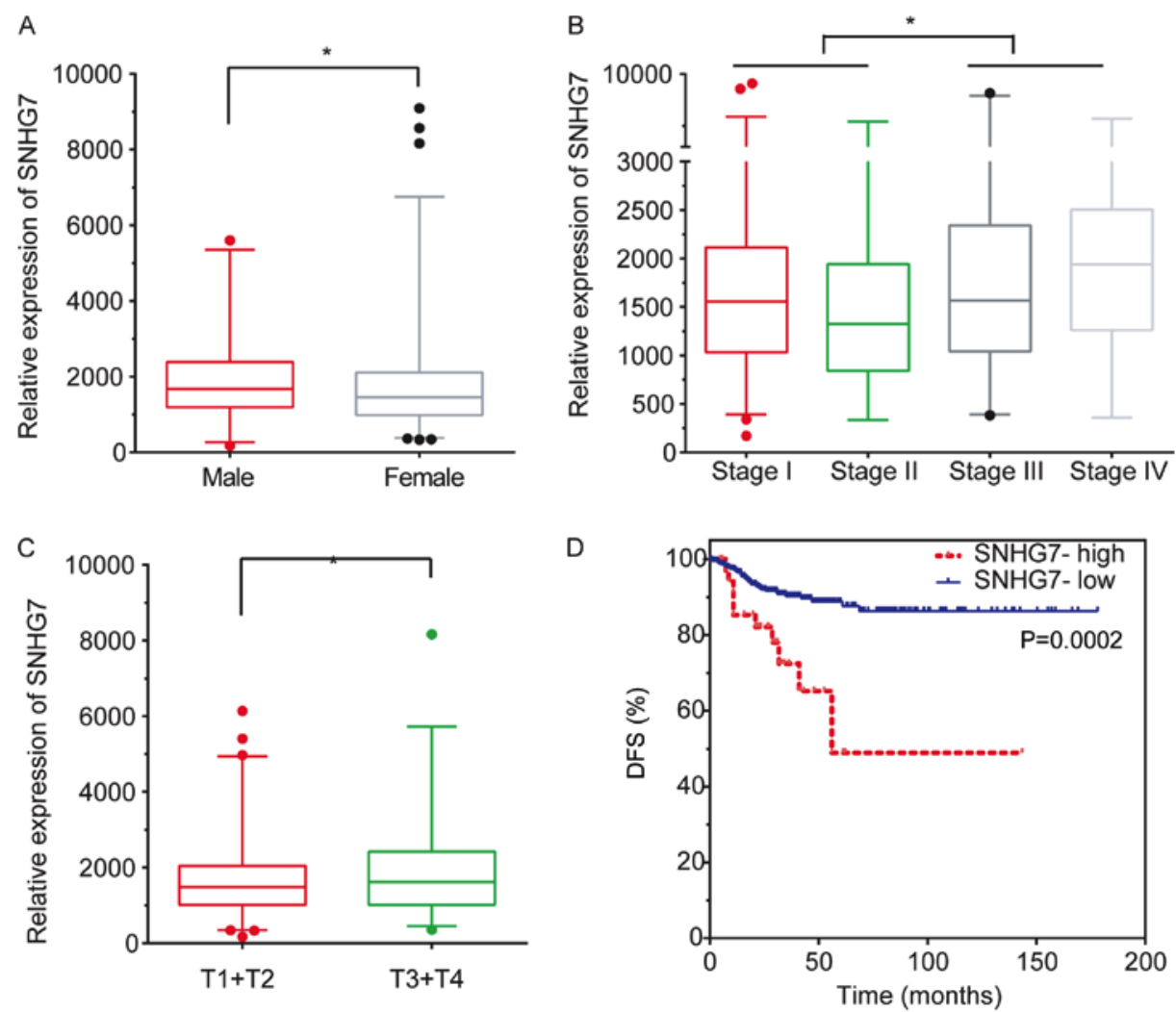

Figure 2. Upregulation of SNHG7 is associated with clinical features of thyroid cancer. (A) SNHG7 was downregulated in female patients with thyroid cancer compared with male patients with thyroid cancer. (B) SNHG7 expression was higher in patients with stages III and IV compared with patients with stages I and II. (C) SNHG7 expression was significantly upregulated in the advanced T3 and T4 stages compared with early T1 and T2 stages. (D) Upregulation of SNHG7 was significantly associated with shorter DFS times in patients with thyroid cancer and high expression level of SNHG7 compared with those with a lower expression level (median DFS time, 56.11 vs. 67.22 months, respectively). "P<0.05, as indicated. SNHG7, small nucleolar RNA host gene 7; T, tumor; DFS, disease-free survival.

from adjacent healthy tissues from patients with THCA) (23), and GSE33630 (obtained from healthy controls) $(24,25)$, were analyzed. SNHG7 expression levels were increased in THCA samples compared with normal samples $(\mathrm{P}<0.001$ and $\mathrm{P}<0.05$; Fig. 1C and D).

High expression of SNHG7 is associated with progression of thyroid cancer. Subsequently, the association between SNHG7 expression levels and clinicopathological features, including age, sex, grade, $\mathrm{T}$ and $\mathrm{N}$ stage and the recurrence status, were evaluated. The analysis revealed that SNHG7 was downregulated in female patients with THCA compared with male patients with THCA $(\mathrm{P}<0.05$; Fig. $2 \mathrm{~A})$. Furthermore, SNHG7 expression was increased in patients with stage III and stage IV compared with patients with stage I $(\mathrm{P}<0.05)$ and stage II $(\mathrm{P}<0.01)$ THCA $(\mathrm{P}<0.05$; Fig. 2B).

SNHG7 expression was revealed to be significantly upregulated in stage $\mathrm{T} 3$ and $\mathrm{T} 4$ patients compared with stage $\mathrm{T} 1$ and $\mathrm{T} 2$ patients $(\mathrm{P}<0.05$; Fig. $2 \mathrm{C})$. This analysis demonstrated that an increased lncRNA SNHG7 expression level was associated with advanced pathology stages in THCA.

IncRNA SNHG7 is associated with poor prognosis in THCA. To further investigate the clinical significance of lncRNA SNHG7 in THCA, SNHG7 expression and survival time in patients with THCA was subsequently analyzed. The results obtained demonstrated that a higher expression level of SNHG7 was closely associated with shorter DFS time in thyroid cancer $(\mathrm{P}<0.001$; median DFS time, 56.11 and 67.22 months for the high and low-expression groups, respectively; Fig. 2D), suggesting that SNHG7 may serve as a biomarker in THCA.

Construction of SNHG7 mediated PPI networks in THCA. By using the 'guilt-by-association' approach, the potential roles of SNHG7 should be similar with its downstream targets. Thus, we predicted potential roles of SNHG7 using SNHG7-regulating mRNAs. Co-expression analysis for SNHG7 in THCA was subsequently performed. The absolute values of Pearson's correlation coefficient $\geq 0.3$ were selected as the cut-off to identify reliable SNHG7-mRNA pairs.

In addition, SNHG7 mediated PPInetworks in thyroidcancer were also constructed. The top two positively co-expressed hub modules are presented in Fig. 3, and the top two negatively co-expressed hub modules are presented in Fig. 4. Positive-related module 1 contained 97 nodes and 4,438 edges, whereas positive-related module 2 contained 51 nodes and 697 edges. Furthermore, negative-related module 1 contained 24 nodes and 276 edges, and negative-related module 2 contained 35 nodes and 182 edges.

GO and KEGG pathway analysis of SNHG7 mediated hub PPI networks in THCA. GO and KEGG pathway analysis of SNHG7 mediated hub PPI networks in thyroid cancer were 
A

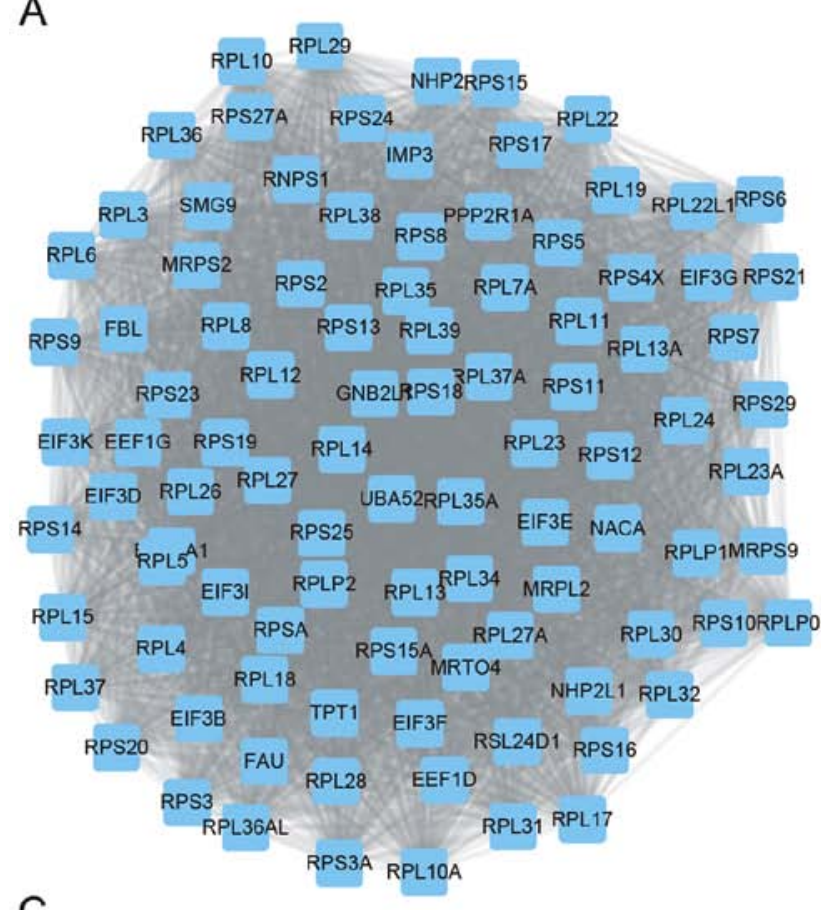

C

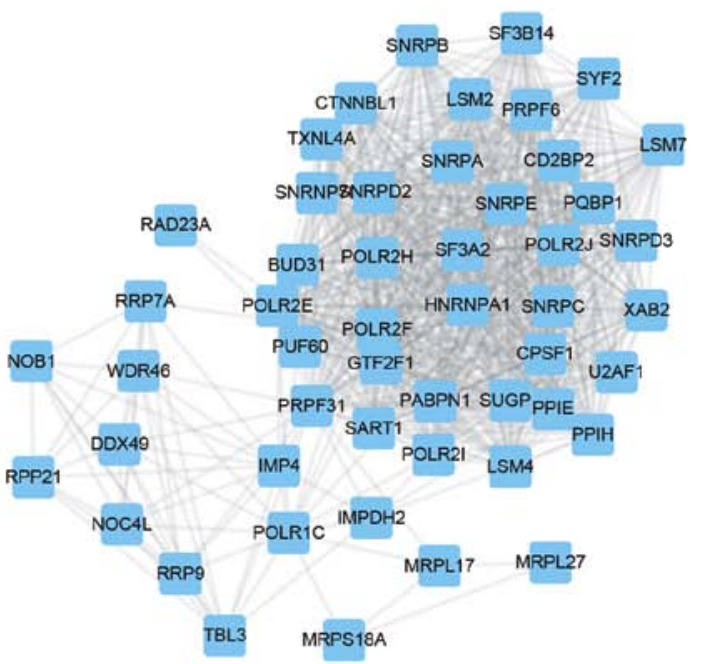

B

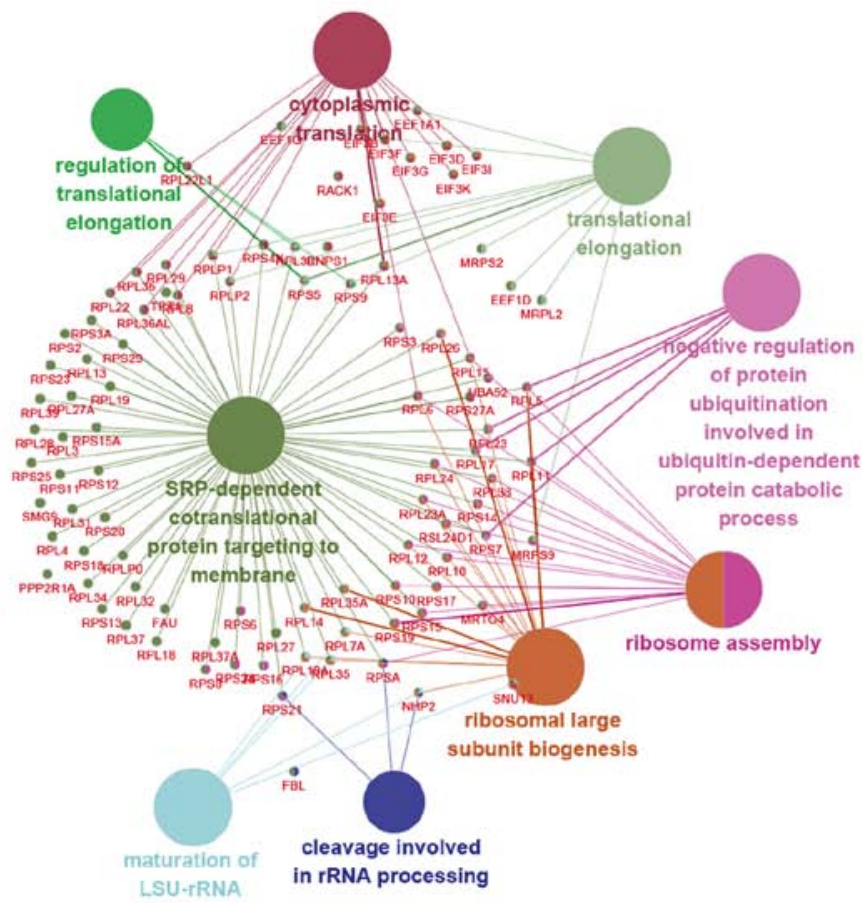

D

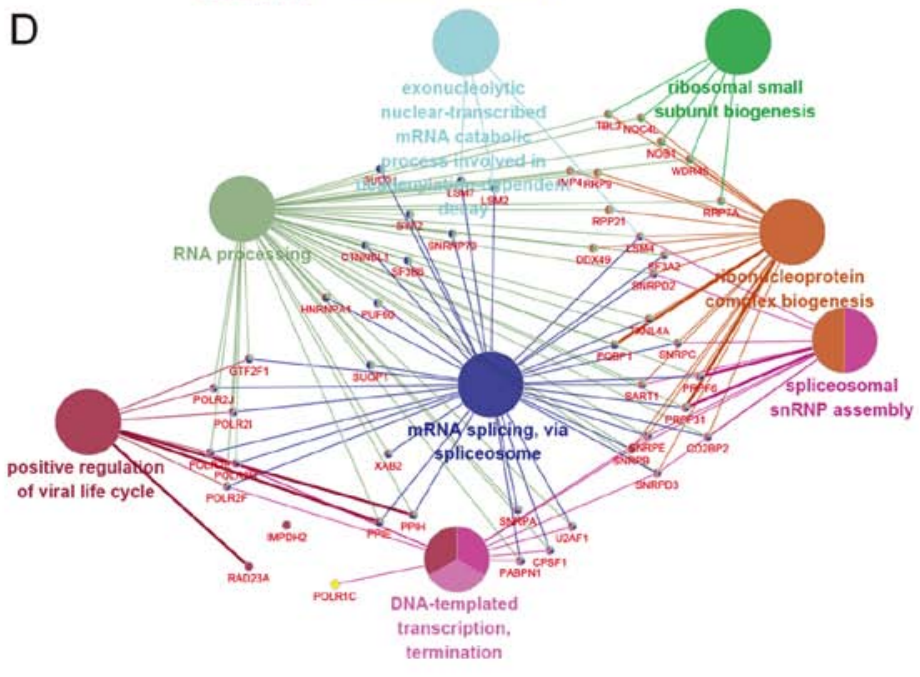

Figure 3. PPI networks and GO and KEGG pathway analyses for the top two positively co-expressed hub modules of SNHG7 in thyroid cancer. (A) Positively co-expressed PPI hub module 1 contained 97 nodes and 4,438 edges. (B) GO and KEGG pathway analyses for positively co-expressed hub module 1. (C) Positively co-expressed PPI hub module 2 contained 51 nodes and 697 edges. (D) GO and KEGG pathway analyses for positively co-expressed hub module 2. PPI, protein-protein interaction; SNHG7, small nucleolar RNA host gene 7; GO, Gene Ontology; KEGG, Kyoto Encyclopedia of Genes and Genomes.

subsequently performed using Cytoscape's ClueGo plug-in. Significant biological processes and pathways $(\mathrm{P}<0.05)$ are presented in the present study. The results revealed that SNHG7 positive-related module 1 was involved in regulating cytoplasmic translation, translation elongation, signal-recognition particle-dependent co-translational protein targeting to membrane and ribosome assembly, whereas SNHG7 positive-related module 2 was associated with positive regulation of the viral life cycle, RNA processing and mRNA splicing.

Furthermore, SNHG7 negative-related module 1 was revealed to be involved in regulating histone ubiquitination and ubiquitin-mediated proteolysis, and SNHG7 negative-related module 1 was revealed to be involved in endoplasmic reticulum (ER)-to-Golgi vesicle-mediated transport, sister chromatid cohesion and DNA damage checkpoint regulation.
Knockdown of SNHG7 inhibits THCA cell proliferation and cell cycle in vitro. In order to further validate the roles of SNHG7 in thyroid cancer, a loss-of-function assay was performed in the CAL62 and SW579 cell lines. As shown Fig. 5A and C, transfection with siSNHG7 significantly decreased the mRNA expression of SNHG7 in CAL62 and SW579 cells compared with the negative control $(\mathrm{P}<0.05)$. The CCK-8 assay was performed to evaluate the effect of SNHG7 knockdown on cell proliferation. As presented in Fig. 5, knockdown of SNHG7 significantly inhibited proliferation in CAL62 (P<0.05; Fig. 5B) and SW579 (P<0.01; Fig. 5D) cells after 4 days.

As abnormal cell cycle progression is a hallmark of cancer $(29,30)$, the role of SNHG7 knockdown in regulating the cell cycle was investigated by flow cytometry in CAL62 and 

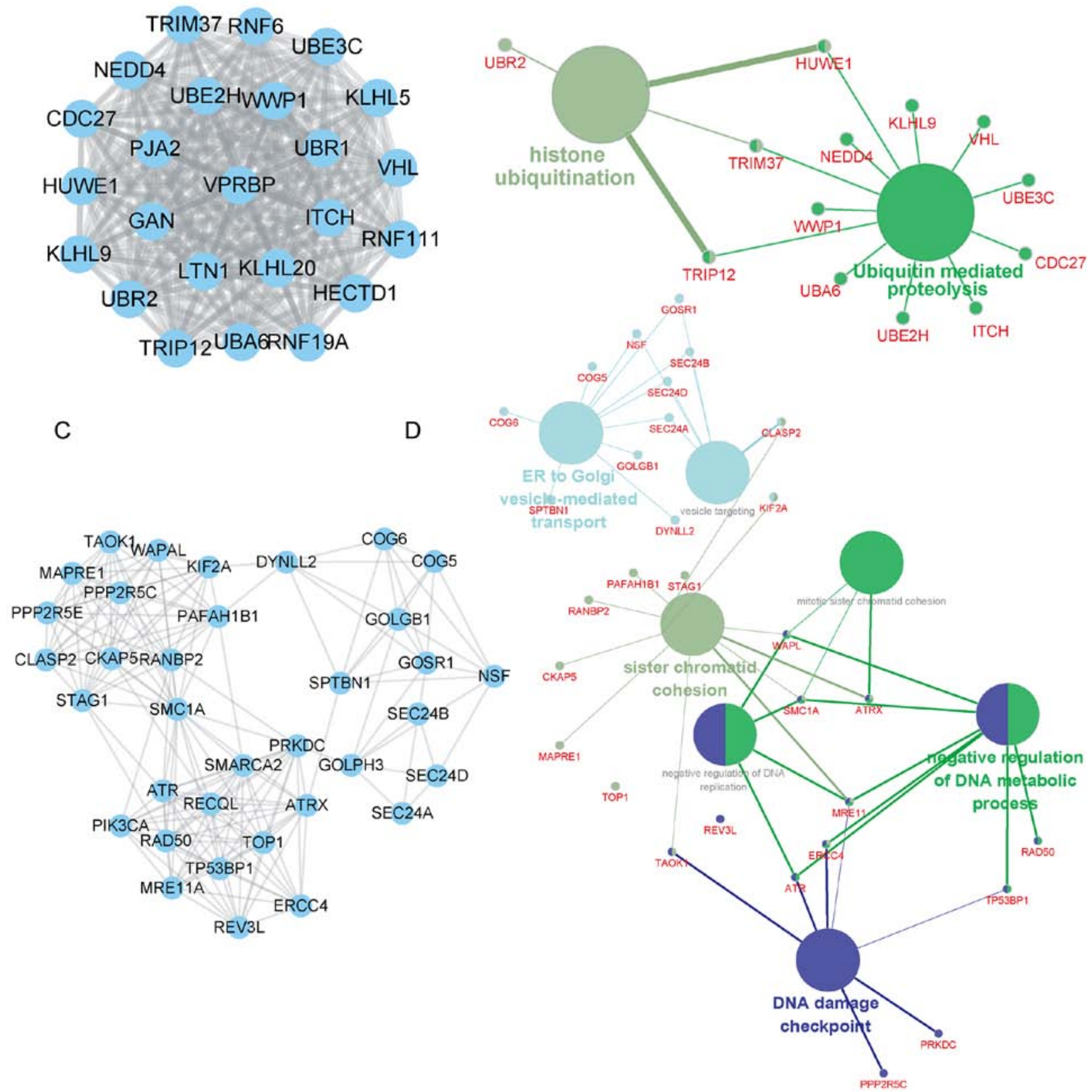

Figure 4. Bioinformatics analyses for the top two negatively co-expressed hub modules of SNHG7 in thyroid cancer. (A) Negatively co-expressed PPI hub module 1 contained 24 nodes and 276 edges. (B) GO and KEGG pathway analyses for negatively co-expressed hub module 1 . (C) Module 2 contained 35 nodes and 182 edges. (D) GO and KEGG pathway analyses for the negatively co-expressed hub module 2. PPI, protein-protein interaction; GO, Gene Ontology; KEGG, Kyoto Encyclopedia of Genes and Genomes; SNHG7, small nucleolar RNA host gene 7.

SW579 cells (Fig. 5E-H). SNHG7 knockdown increased the percentage of cells in the $\mathrm{G}_{1}$ phase and decreased the percentage of cells in the $\mathrm{S}$ phase compared with control cells. Furthermore, SNHG7 knockdown decreased the percentage of cells in $\mathrm{G}_{2}$ phase in the CAL62 cells compared with control cells, but no significant difference was observed in the SW579 cells $(\mathrm{P}<0.01)$.

\section{Discussion}

An increasing number of studies have demonstrated the lncRNAs serve important roles in various types of human cancer, including prostate $(31,32)$, breast $(33,34)$, lung $(35,36)$ and THCA (37). Specific IncRNAs have been reported to be involved in THCA progression. For example, Pvt1 oncogene was the first lncRNA revealed to be associated with the regulation of THCA cell proliferation by increasing the expression of thyroid-stimulating hormone receptor (38). Xu et al (39) observed that the IncRNAs ENST00000537266 and ENST00000426615 served roles in papillary THCA growth and metastasis. H19 imprinted maternally expressed transcript was found to be a ceRNA by 'sponging' miR-17-5p to regulate the expression of YES proto-oncogene 1 Src family tyrosine kinase in THCA (40). The present study revealed, to the best of the best of the authors' knowledge for the first time, that lncRNA SNHG7 is upregulated in, and associated with the prognosis of, THCA. 


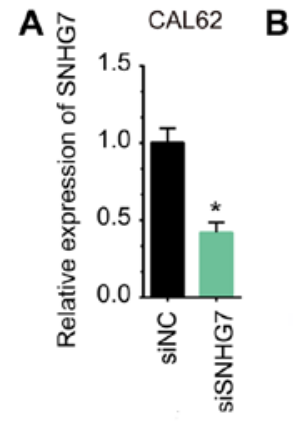

E
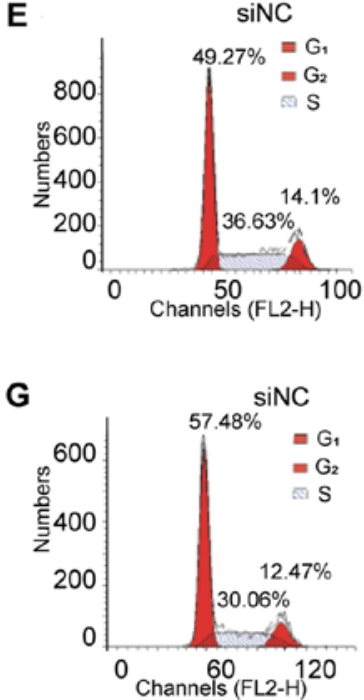
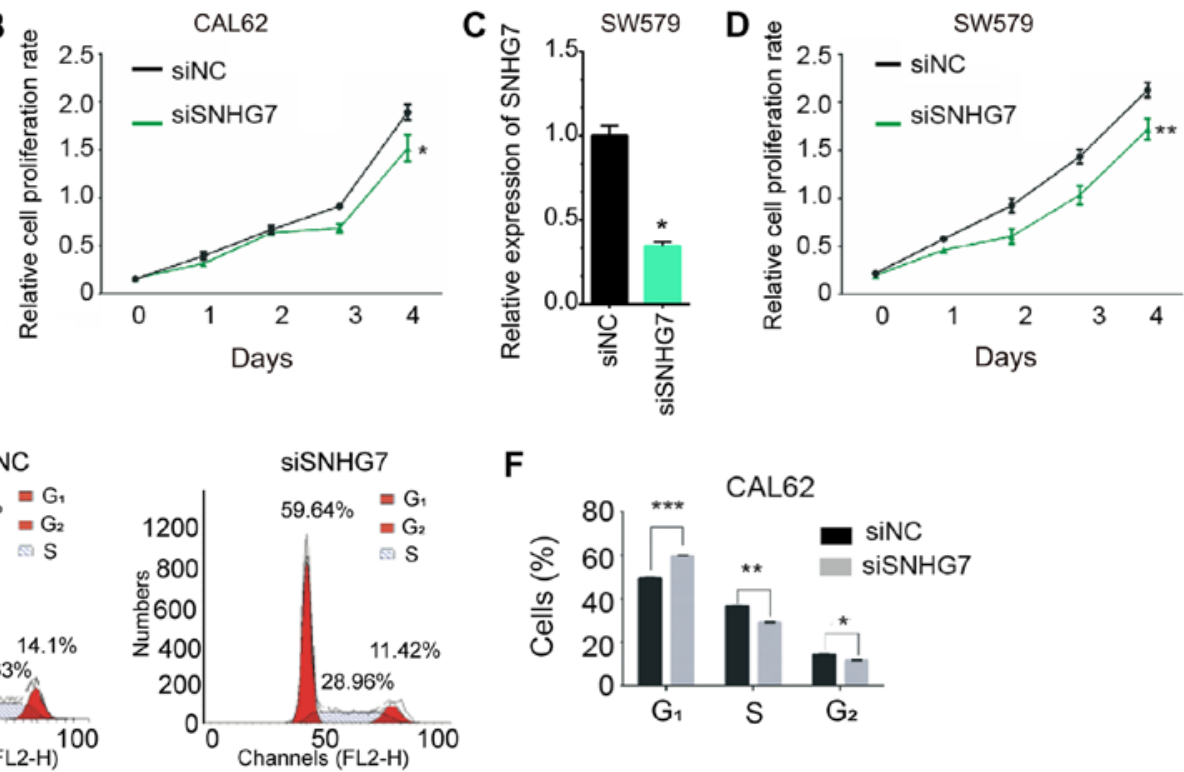

$\mathbf{F}$
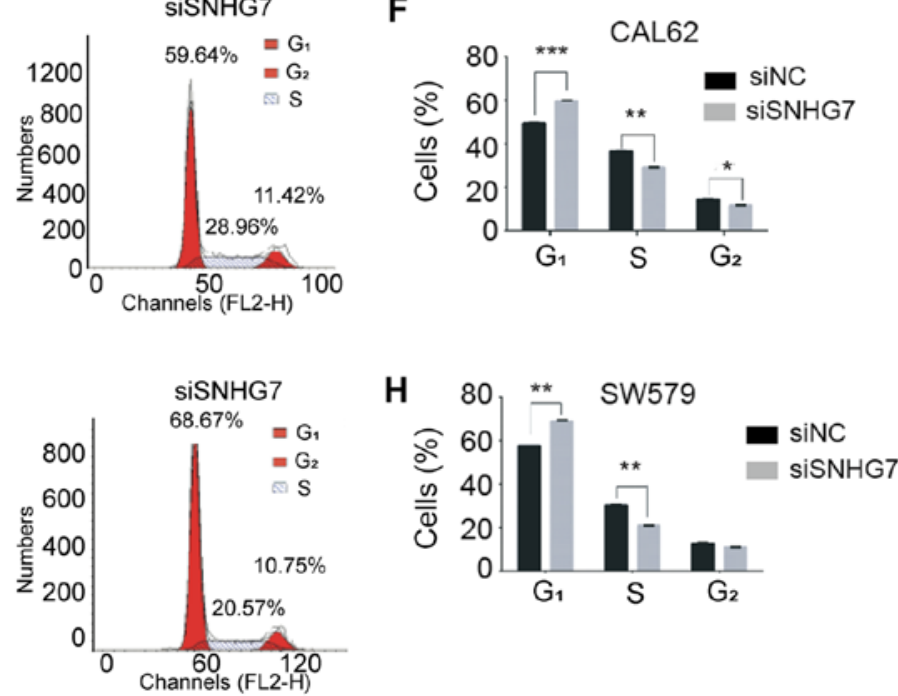

Figure 5. Knockdown of SNHG7 inhibited thyroid cancer cell proliferation and cell cycle. (A) The mRNA expression of SNHG7 was significant decreased by siSNHG7 in CAL62 cells. (B) Knockdown of SNHG7 inhibited cell proliferation compared with control cells in CAL62 cells. (C) The mRNA expression of SNHG7 was significant decreased by siSNHG7 in SW579 cells. (D) Knockdown of SNHG7 inhibited cell proliferation compared with control cells in SW579 cells. (E) Knockdown of SNHG7 in CAL62 cells increased the percentage of cells in the $\mathrm{G}_{1}$ phase and decreased the percentage of cells in $S$ and $\mathrm{G}_{2}$ phases compared with control cells. (F) Statistical histogram of cell cycle distribution after SNHG7 silencing in CAL62. (G) Knockdown of SNHG7 in SW579 cells increased the percentage of cells in $\mathrm{G}_{1}$ phase and decreased the percentage of cells in $\mathrm{S}$ phase compared with control cells. (H) Statistical histogram of cell cycle distribution after SNHG7 silencing in SW579 cells. ${ }^{*} \mathrm{P}<0.05 ;{ }^{* *} \mathrm{P}<0.01 ;{ }^{* * *} \mathrm{P}<0.001$, vs. siNC. SNHG7, small nucleolar RNA host gene 7; si, small interfering; $\mathrm{NC}$, negative control.

SNHG7 is a novel lncRNA that has been reported to have an oncogenic role in different types of human cancer. SNHG7 is upregulated in esophageal (41) and prostate cancer (42), glioblastoma (43), non-small cell lung (44) and gastric cancer (19). However, the roles of SNHG7 in THCA have yet to be elucidated. In the present study, analysis of the TCGA dataset revealed that SNHG7 was upregulated in THCA tissues compared with normal samples. Notably, high expression of SNHG7 was revealed to be associated with the progression of THCA. SNHG7 was upregulated in advanced-stage (stages III and IV) compared with the low-stage (stages I and II) THCA samples. Furthermore, the present study revealed, to the best of best of the authors' knowledge for the first time, that higher expression levels of SNHG7 were closely associated with shorter overall survival and DFS times in THCA. Taken together, these results demonstrated that SNHG7 may serve as a biomarker for THCA.

Co-expression analysis has been widely used to identify potential roles of lncRNAs. For example, Chen et al (45) constructed an lncRNA-mRNA co-expression network to reveal the roles of IncRNAs in zebra fish. In the current study, an SNHG7-mediated co-expression network in thyroid cancer was constructed. The four top hub modules mediated by SNHG7 were identified in THCA. The present analysis revealed that SNHG7 is associated with 'translation', 'viral life cycle', 'RNA processing', 'mRNA splicing', 'histone ubiquitination', 'ER-to-Golgi vesicle-mediated transport', 'sister chromatid cohesion' and 'DNA damage checkpoint regulation'. In order to validate the roles of SNHG7 in THCA, an in vitro loss-of-function assay was performed, and the results obtained revealed that SNHG7 knockdown leads to a marked inhibition of cell proliferation and cell cycle progression in THCA cell lines. Taken together, the results obtained in the current study suggest that SNHG7 is an oncogene in THCA.

In conclusion, the present study has demonstrated that SNHG7 was markedly upregulated in THCA samples compared with control samples through an analysis of TCGA datasets. SNHG7 expression levels were increased in advanced-stage compared with early-stage THCA samples. Additionally, a higher expression level of SNHG7 was associated with shorter survival times compared with a lower expression level. The results obtained in the current study suggest that SNHG7 may be used to predict the prognosis of patients with THCA. The present study suggested that SNHG7 may serve as a new therapeutic and prognostic target for THCA. 


\section{Acknowledgements}

Not applicable.

\section{Funding}

No funding was received.

\section{Availability of data and materials}

All data generated or analyzed during this study are included in this published article.

\section{Authors' contributions}

LC and LJZ designed the study. LC, LJZ and JZ developed the methodology. LC, LJZ and JZ analyzed and interpreted the data, and wrote and revised the manuscript. All authors read and approved the final manuscript.

\section{Ethics approval and consent to participate}

Not applicable.

\section{Patient consent for publication}

Not applicable.

\section{Competing interests}

The authors declare that they have no competing interests.

\section{References}

1. Chen AY, Jemal A and Ward EM: Increasing incidence of differentiated thyroid cancer in the United States, 1988-2005. Cancer 115: 3801-3807, 2009.

2. Pellegriti G, Frasca F, Regalbuto C, Squatrito S and Vigneri R: Worldwide increasing incidence of thyroid cancer: Update on epidemiology and risk factors. J Cancer Epidemiol 2013: 965212, 2013.

3. Huang P, Mao LF, Zhang ZP, Lv WW, Feng XP, Liao HJ, Dong C, Kaluba B, Tang XF and Chang S: Down-Regulated miR-125a-5p promotes the reprogramming of glucose metabolism and cell malignancy by increasing levels of CD147 in thyroid cancer. Thyroid 28: 613-623, 2018.

4. Choi D, Ramu S, Park E, Jung E, Yang S, Jung W, Choi I, Lee S, Kim KE, Seong YJ, et al: Aberrant activation of notch signaling inhibits PROX1 activity to enhance the malignant behavior of thyroid cancer cells. Cancer Res 76: 582-593, 2016.

5. Prensner JR and Chinnaiyan AM: The emergence of lncRNAs in cancer biology. Cancer Discov 1: 391-407, 2011.

6. Wang F, Yuan JH, Wang SB, Yang F, Yuan SX, Ye C, Yang N, Zhou WP, Li WL, Li W and Sun SH: Oncofetal long noncoding RNA PVT1 promotes proliferation and stem cell-like property of hepatocellular carcinoma cells by stabilizing NOP2 Hepatology 60: 1278-1290, 2014.

7. Cao C, Sun J, Zhang D, Guo X, Xie L, Li X, Wu D and Liu L: The long intergenic noncoding RNA UFC1, a target of MicroRNA 34a, interacts with the mRNA stabilizing protein HuR to increase levels of $\beta$-catenin in HCC cells. Gastroenterology 148: 415-426, 2015.

8. Engreitz JM, Pandya-Jones A, McDonel P, Shishkin A, Sirokman K, Surka C, Kadri S, Xing J, Goren A, Lander ES, et al: The Xist lncRNA exploits three-dimensional genome architecture to spread across the X chromosome. Science 341: 1237973, 2013.

9. Yang F, Deng X, Ma W, Berletch JB, Rabaia N, Wei G, Moore JM Filippova GN, Xu J, Liu Y, et al: The lncRNA Firre anchors the inactive $\mathrm{X}$ chromosome to the nucleolus by binding CTCF and maintains H3K27me3 methylation. Genome Biol 16: 52, 2015.
10. Yoon JH, Abdelmohsen K and Gorospe M: Posttranscriptional gene regulation by long noncoding RNA. J Mol Biol 425: 3723-3730, 2013.

11. Sun W, Lan X, Zhang H, Wang Z, Dong W, He L, Zhang T, Zhang P, Liu J and Qin Y: NEAT1_2 functions as a competing endogenous RNA to regulate ATAD2 expression by sponging microRNA-106b-5p in papillary thyroid cancer. Cell Death Dis 9: 380,2018

12. Chen C, Zhou L, Wang H, Chen J, Li W, Liu W, Shen M, Liu H and Fu X: Long noncoding RNA CNALPTC1 promotes cell proliferation and migration of papillary thyroid cancer via sponging miR-30 family. Am J Cancer Res 8: 192-206, 2018.

13. Zhang R, Hardin H, Chen J, Guo Z and Lloyd RV: Non-coding RNAs in thyroid cancer. Endocr Pathol 27: 12-20, 2016.

14. Yang G, Lu X and Yuan L: LncRNA: A link between RNA and cancer. Biochim Biophys Acta 1839: 1097-1109, 2014.

15. Huang JK, Ma L, Song WH, Lu BY, Huang YB, Dong HM, Ma XK, Zhu ZZ and Zhou R: LncRNA-MALAT1 Promotes angiogenesis of thyroid cancer by modulating tumor-associated macrophage FGF2 protein secretion. J Cell Biochem 118: 4821-4830, 2017.

16. Li Q, Li H, Zhang L, Zhang C, Yan W and Wang C: Identification of novel long non-coding RNA biomarkers for prognosis prediction of papillary thyroid cancer. Oncotarget 8: 46136-46144, 2017.

17. Guo LJ, Zhang S, Gao B, Jiang Y, Zhang XH, Tian WG, Hao S, Zhao JJ, Zhang G, Hu CY, et al: Low expression of long non-coding RNA GAS5 is associated with poor prognosis of patients with thyroid cancer. Exp Mol Pathol 102: 500-504, 2017.

18. She K, Huang J, Zhou H, Huang T, Chen $G$ and He J: lncRNA-SNHG7 promotes the proliferation, migration and invasion and inhibits apoptosis of lung cancer cells by enhancing the FAIM2 expression. Oncol Rep 36: 2673-2680, 2016.

19. Wang MW, Liu J, Liu Q, Xu QH, Li TF, Jin S and Xia TS: LncRNA SNHG7 promotes the proliferation and inhibits apoptosis of gastric cancer cells by repressing the P15 and P16 expression. Eur Rev Med Pharmacol Sci 21: 4613-4622, 2017.

20. Gao J, Aksoy BA, Dogrusoz U, Dresdner G, Gross B, Sumer SO, Sun Y, Jacobsen A, Sinha R, Larsson E, et al: Integrative analysis of complex cancer genomics and clinical profiles using the cBioPortal. Sci Signal 6: pl1, 2013.

21. Cerami E, Gao J, Dogrusoz U, Gross BE, Sumer SO, Aksoy BA, Jacobsen A, Byrne CJ, Heuer ML, Larsson E, et al: The cBio cancer genomics portal: An open platform for exploring multidimensional cancer genomics data. Cancer Discov 2: 401-404, 2012.

22. Edge SB, Byrd DR, Compton CC, Fritz AG, Greene FL and Trotti A: AJCC cancer staging manual 7th edition, NY Springer, 2010.

23. Barros-Filho MC, Marchi FA, Pinto CA, Rogatto SR and Kowalski LP: High diagnostic accuracy based on CLDN10, HMGA2, and LAMB3 transcripts in papillary thyroid carcinoma. J Clin Endocrinol Metab 100: E890-E899, 2015.

24. Tomas G, Tarabichi M, Gacquer D, Hebrant A, Dom G, Dumont JE, Keutgen X, Fahey TR, Maenhaut C and Detours V: A general method to derive robust organ-specific gene expression-based differentiation indices: Application to thyroid cancer diagnostic. Oncogene 31: 4490-4498, 2012.

25. Dom G, Tarabichi M, Unger K, Thomas G, OczkoWojciechowska M, Bogdanova T, Jarzab B, Dumont JE, Detours $\mathrm{V}$ and Maenhaut C: A gene expression signature distinguishes normal tissues of sporadic and radiation-induced papillary thyroid carcinomas. Br J Cancer 107: 994-1000, 2012.

26. Szklarczyk D, Gable AL, Lyon D, Junge A, Wyder S, HuertaCepas J, Simonovic M, Doncheva NT, Morris JH, Bork P, et al: STRING v11: Protein-protein association networks with increased coverage, supporting functional discovery in genome-wide experimental datasets. Nucleic Acids Res 47: D607-D613, 2019.

27. Bader GD and Hogue CW: An automated method for finding molecular complexes in large protein interaction networks. Bmc Bioinformatics 4: 2, 2003.

28. Livak KJ and Schmittgen TD: Analysis of relative gene expression data using real-time quantitative PCR and the 2(-Delta Delta C(T)) method. Methods 25: 402-408, 2001.

29. Williams GH and Stoeber K: The cell cycle and cancer. J Pathol 226: 352-364, 2012.

30. Kastan MB and Bartek J: Cell-cycle checkpoints and cancer. Nature 432: 316-323, 2004.

31. Misawa A, Takayama KI and Inoue S: Long non-coding RNAs and prostate cancer. Cancer Sci 108: 2107-2114, 2017. 
32. Wan X, Huang W, Yang S, Zhang Y, Pu H, Fu F, Huang Y, Wu H, $\mathrm{Li} \mathrm{T}$ and $\mathrm{Li} \mathrm{Y}$ : Identification of androgen-responsive lncRNAs as diagnostic and prognostic markers for prostate cancer. Oncotarget 7: 60503-60518, 2016.

33. Kumar M, DeVaux RS and Herschkowitz JI: Molecular and cellular changes in breast cancer and new roles of lncRNAs in breast cancer initiation and progression. Prog Mol Biol Transl Sci 144: 563-586, 2016.

34. Li T, Liu Y, Xiao H and Xu G: Long non-coding RNA TUG1 promotes cell proliferation and metastasis in human breast cancer. Breast Cancer 24: 535-543, 2017.

35. Park SM, Choi EY, Bae DH, Sohn HA, Kim SY and Kim YJ: The LncRNA EPEL promotes lung cancer cell proliferation through E2F target activation. Cell Physiol Biochem 45: 1270-1283, 2018.

36. Tao H, Yang JJ, Zhou X, Deng ZY, Shi KH and Li J: Emerging role of long noncoding RNAs in lung cancer: Current status and future prospects. Respir Med 110: 12-19, 2016.

37. Kim D, Lee WK, Jeong S, Seol MY, Kim H, Kim KS, Lee EJ, Lee J and Jo YS: Upregulation of long noncoding RNA LOC100507661 promotes tumor aggressiveness in thyroid cancer. Mol Cell Endocrinol 431: 36-45, 2016.

38. Zhou Q, Chen J, Feng J and Wang J: Long noncoding RNA PVT1 modulates thyroid cancer cell proliferation by recruiting EZH2 and regulating thyroid-stimulating hormone receptor (TSHR). Tumour Biol 37: 3105-3113, 2016.

39. Xu B, Shao Q, Xie K, Zhang Y,Dong T, Xia Y and Tang W: The long non-coding RNA ENST00000537266 and ENST00000426615 influence papillary thyroid cancer cell proliferation and motility. Cell Physiol Biochem 38: 368-378, 2016.
40. Liu L, Yang J, Zhu X, Li D, Lv Z and Zhang X: Long noncoding RNA H19 competitively binds miR-17-5p to regulate YES1 expression in thyroid cancer. FEBS J 283: 2326-2339, 2016.

41. Xu LJ, Yu XJ, Wei B, Hui HX, Sun Y, Dai J and Chen XF: LncRNA SNHG7 promotes the proliferation of esophageal cancer cells and inhibits its apoptosis. Eur Rev Med Pharmacol Sci 22: 2653-2661, 2018.

42. Qi H, Wen B, Wu Q, Cheng W, Lou J, Wei J, Huang J, Yao X and Weng G: Long noncoding RNA SNHG7 accelerates prostate cancer proliferation and cycle progression through cyclin D1 by sponging miR-503. Biomed Pharmacother 102: 326-332, 2018.

43. Ren J, Yang Y, Xue J, Xi Z, Hu L, Pan SJ and Sun Q: Long noncoding RNA SNHG7 promotes the progression and growth of glioblastoma via inhibition of miR-5095. Biochem Biophys Res Commun 496: 712-718, 2018.

44. She K, Yan H, Huang J, Zhou H and He J: miR-193b availability is antagonized by LncRNA-SNHG7 for FAIM2-induced tumour progression in non-small cell lung cancer. Cell Prolif: Nov 12, 2017 (Epub ahead of print). doi: 10.1111/cpr.12406.

45. Chen W, Zhang X, Li J, Huang S, Xiang S, Hu X and Liu C: Comprehensive analysis of coding-lncRNA gene co-expression network uncovers conserved functional lncRNAs in zebrafish. Bmc Genomics 19 (Suppl 2): S112, 2018.

This work is licensed under a Creative Commons Attribution-NonCommercial-NoDerivatives 4.0 International (CC BY-NC-ND 4.0) License. 